Cite this: Phys. Chem. Chem. Phys., 2013

\title{
Single-crystal X-ray structure analysis of the superionic conductor $\mathrm{Li}_{10} \mathrm{GeP}_{2} \mathrm{~S}_{12}{ }^{\dagger}$
}

15, 11620

Received 10th May 2013,

Accepted 30th May 2013

DOI: $10.1039 / c 3 c p 51985 f$

www.rsc.org/pccp

Tetragonal $\mathrm{Li}_{10} \mathrm{GeP}_{2} \mathrm{~S}_{12}$ (LGPS) is the best solid $\mathrm{Li}$ ion conductor known to date. So far, the structure of the electrolyte was only determined from powder diffraction and Rietveld refinement. Here, we present the first single-crystal structure analysis of the tetragonal LGPS structure. The reported structure is largely verified. However, an additional Li position is clearly identified which might have a significant impact on the Li ion dynamics. All Li positions are partially occupied - a prerequisite for Li superionic conductors - and form a network of interconnected Li diffusion pathways. Therefore, we suggest that Li diffusion in this record solid electrolyte is less anisotropic than previously claimed.

Energy storage is one of the key challenges in the transition from fossil to renewable energy sources. Hereby, Li-ion batteries are considered to play an important role in the future. ${ }^{1}$ The lack of a suitable solid Li electrolyte for Li-ion batteries triggered intense research in the past decade (see, e.g. ref. 2-4). In 2011, the new electrolyte $\mathrm{Li}_{10} \mathrm{GeP}_{2} \mathrm{~S}_{12}$ was reported to combine two desired properties: a high room-temperature conductivity of $12 \mathrm{mS} \mathrm{cm}^{-1}$ and a wide electrochemical window of up to $5 \mathrm{~V}$ against $\mathrm{Li} / \mathrm{Li}^{+}$. Although the latter point is probably due to the formation of a protective interface rather than an intrinsic property of the material, ${ }^{5} \mathrm{Li}_{10} \mathrm{GeP}_{2} \mathrm{~S}_{12}$ or related materials ${ }^{6,7}$ are still promising candidates for future commercial application.

In the literature, the tetragonal structure of $\mathrm{Li}_{10} \mathrm{GeP}_{2} \mathrm{~S}_{12}$ (space group $P 4_{2} / n m c$ (137)) was determined from powder diffraction and Rietveld refinement. The reported structure (see Fig. 1B) consists of negatively charged $\left(\mathrm{PS}_{4}\right)^{3-}$ and $\left(\mathrm{GeS}_{4}\right)^{4-}$ tetrahedra which are surrounded by (mobile) $\mathrm{Li}$ ions for charge compensation. The tetrahedrally coordinated Li1 and Li3 sites give rise to channels for facile Li diffusion along the $c$-axis while the octahedrally coordinated Li2 position between those channels was assumed to be inactive for diffusion. ${ }^{4} \mathrm{MD}$ simulations supported this picture of highly anisotropic diffusivity in LGPS. ${ }^{5,6,8,9}$

\footnotetext{
${ }^{a}$ Max Planck Institute for Solid State Research, Heisenbergstr. 1, 70569 Stuttgart, Germany.E-mail: a.kuhn@fkf.mpg.de, b.lotsch@fkf.mpg.de

${ }^{b}$ Department of Chemistry, Ludwig-Maximilians-Universität München,

Butenandtstr. 5-15, 81377 München, Germany

$\dagger$ Electronic supplementary information (ESI) available. See DOI: 10.1039/c3cp51985f
}

In this communication, we report the first single-crystal structure analysis of the tetragonal LGPS structure. The structure was determined both at room temperature and at $100 \mathrm{~K}$. The study provides significantly more reliable information on the structure than available so far and clearly reveals four Li positions, one of which was not reported from the neutron powder diffraction Rietveld refinement.

Stoichiometric amounts of $\mathrm{Li}_{2} \mathrm{~S}$ (Alfa Aesar, 99.9\%), Ge (Aldrich, 99.999\%), P (Alfa Aesar, 98.9\%), and a slight excess of S (Alfa Aesar, 99.5\%) were mechanically treated in a Fritsch planetary mill for 1 day. The powder was then heated to $1023 \mathrm{~K}$ in an evacuated quartz tube and slowly cooled down to room temperature. The obtained crystalline product is a mixture of $\mathrm{Li}_{10} \mathrm{GeP}_{2} \mathrm{~S}_{12}$ in the desired tetragonal and the orthorhombic modification (a solid solution showing the $\mathrm{Li}_{3} \mathrm{PS}_{4}$ structure). Lowering the synthesis temperature increases the fraction of

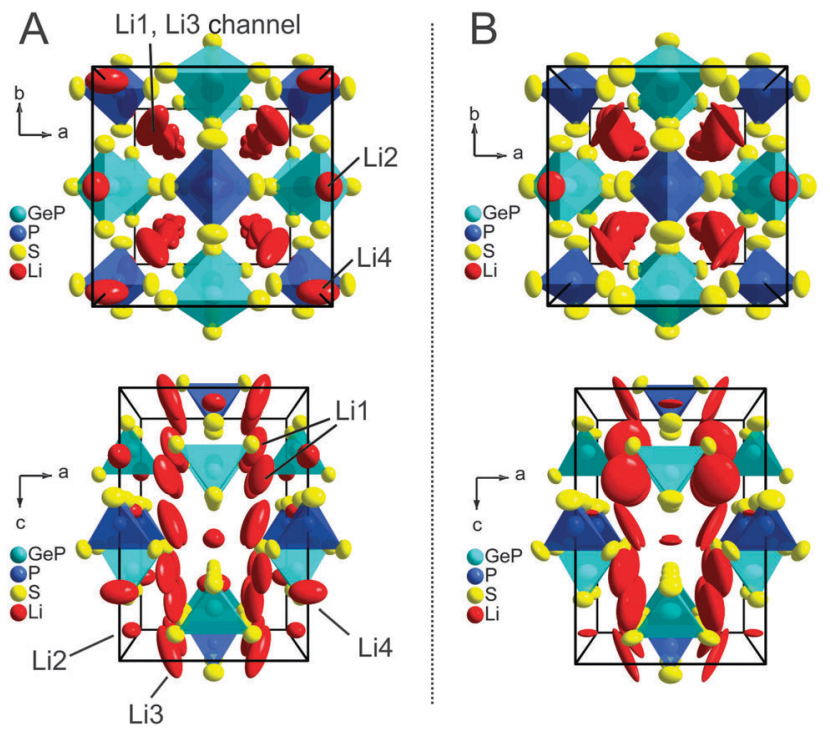

Fig. 1 Unit cell of tetragonal $\mathrm{Li}_{10} \mathrm{GeP}_{2} \mathrm{~S}_{12}$ with thermal ellipsoids ( $p=0.8$ ). (A) Data obtained from single crystal X-ray diffraction (this study), (B) data obtained from neutron powder diffraction Rietveld refinement (ref. 4). 
the tetragonal structure with, however, smaller crystallites unsuitable for single-crystal X-ray diffraction.

For single crystal X-ray diffraction, several slightly yellowish transparent crystals were picked from the reaction product and mounted on sealed glass capillaries. One crystal of the desired tetragonal modification suitable for the single crystal diffraction study was selected. After the diffraction measurements, SEM-EDX was used to confirm the elemental composition. The expected ratio $\mathrm{Ge}: \mathrm{P}: \mathrm{S}=1: 2: 12$ as expected from the stoichiometric formula was obtained within experimental error. All manipulations were carried out under inert conditions, i.e. in an argon-filled glove box or under dry petroleum (crystal picking).

The X-ray single-crystal diffraction data were collected at room temperature and at $100 \mathrm{~K}$ using a SMART-APEX-I CCD X-ray diffractometer (Bruker AXS) with a Cryostream $700^{\text {Plus }}$ cooling device (Oxford Cryosystems, Oxford, UK, 80-500 K) working with graphitemonochromated Mo- $\mathrm{K}_{\alpha}$ radiation. The integration of the reflections was performed using the SAINT software (Bruker). The structure was solved using direct methods and refined by least-squares fitting using the SHELXTL program. ${ }^{11}$

The crystal data and refinement details of the single crystal diffraction measured at room temperature (r.t.) and $100 \mathrm{~K}$ are presented in Table 1. Atomic coordinates and their occupancies are given in Table 2, the anisotropic displacement parameters are shown in Table 3. For comparison, data obtained from Rietveld refinement of neutron powder diffraction data $(300 \mathrm{~K})^{4}$ are displayed in Tables 2 and 3 as well. First of all, the reported structure model of $\mathrm{Li}_{10} \mathrm{GeP}_{2} \mathrm{~S}_{12}$ (ref. 4) is largely corroborated by our single crystal X-ray diffraction study. The space group $\mathrm{P4}_{2} / \mathrm{nmc}$ is verified and the cell parameters of our room-temperature measurement $(a=8.7187 \AA, c=12.6385 \AA)$ are similar to the reported ones $(a=8.69407 \AA, c=12.5994 \AA)$. The unit cell content is very similar, as well.

The differences concerning the Ge, $\mathrm{P}$, and $\mathrm{S}$ sites are in the low percent range. However, more significant differences are revealed concerning the Li positions: (i) most importantly, we clearly find a fourth $\mathrm{Li}$ site, $\mathrm{Li} 4$, which is occupied by $\approx 70 \%$;

Table 1 Crystallographic data for $\mathrm{Li}_{10} \mathrm{GeP}_{2} \mathrm{~S}_{12}$ (obtained from single crystal X-ray diffraction data)

\begin{tabular}{lll}
\hline $\mathrm{Li}_{10} \mathrm{GeP}_{2} \mathrm{~S}_{12}$ & & \\
\hline Temperature & $298 \mathrm{~K}$ & $100 \mathrm{~K}$ \\
Space group & $P 4_{2} / n m c: 1(137: 1)$ & $P 4_{2} / n m c: 1(137: 1)$ \\
Lattice constants & $a=8.7187 \AA$ & $a=8.6521 \AA$ \\
& $c=12.6385 \AA$ & $a=12.5816 \AA$ \\
Cell volume & $V=960.72 \AA^{3}$ & $V=941.84 \AA^{3}$ \\
Calc. density & $\rho=2.035 \mathrm{~g} \mathrm{~cm}^{-3}$ & $\rho=2.076 \mathrm{~g} \mathrm{~cm}^{-3}$ \\
Radiation & $\mathrm{Mo} \mathrm{K}_{\alpha}$ & $\mathrm{Mo} \mathrm{K} \alpha$ \\
Max. $\Theta \Theta$ & $61.18^{\circ}$ & $61.29^{\circ}$ \\
Index range & $-12 \leq h \leq 12$ & $-12 \leq h \leq 12$ \\
& $-12 \leq k \leq 12$ & $-12 \leq k \leq 12$ \\
Total reflections & $-17 \leq l \leq 17$ & $-17 \leq l \leq 17$ \\
Unique reflections & 10834 & 10274 \\
$R_{\text {int }}$ & 829 & 820 \\
$R_{1}(>4 \sigma)$ & 0.065 & 0.047 \\
$w R_{2}(>4 \sigma)$ & 0.046 & 0.036 \\
GooF & 0.112 & 0.088 \\
Deposition no. ${ }^{10}$ & 1.171 & 1.154 \\
& $\mathrm{CSD}-425992$ & $\mathrm{CSD}-425993$
\end{tabular}

Table 2 Atomic coordinates and occupation factors for $\mathrm{Li}_{10} \mathrm{GeP}_{2} \mathrm{~S}_{12}$ obtained from single crystal $\mathrm{X}$-ray diffraction at room temperature (r.t.) and $100 \mathrm{~K}$ (I.t.) in comparison with literature data obtained from neutron powder diffraction at room temperature $(\mathrm{lit})^{4}$

\begin{tabular}{|c|c|c|c|c|c|c|}
\hline Atom site & & & $x$ & $y$ & $z$ & Occ. \\
\hline \multirow[t]{3}{*}{ Li1 } & $16 \mathrm{~h}$ & lit & $0.2563(5)$ & $0.2718(3)$ & $0.1832(3)$ & $0.691(5)$ \\
\hline & & r.t. & $0.267(3)$ & $0.273(3)$ & $0.194(2)$ & $0.466(18)$ \\
\hline & & 1.t. & $0.257(2)$ & $0.2699(19)$ & $0.1869(16)$ & $0.474(16)$ \\
\hline \multirow{3}{*}{$\mathrm{Li} 2$} & $4 \mathrm{~d}$ & lit & 0 & $1 / 2$ & $0.9446(2)$ & 1.000 \\
\hline & & r.t. & 0 & $1 / 2$ & $0.9454(12)$ & $0.86(6)$ \\
\hline & & l.t. & 0 & $1 / 2$ & $0.9469(9)$ & $0.89(5)$ \\
\hline \multirow[t]{3}{*}{ Li3 } & $8 f$ & lit & $0.2463(5)$ & $=x(\mathrm{Li} 3)$ & 0 & $0.643(5)$ \\
\hline & & r.t. & $0.248(2)$ & $=x(\mathrm{Li} 3)$ & 0 & $0.74(5)$ \\
\hline & & l.t. & $0.2471(15)$ & $=x(\mathrm{Li} 3)$ & 0 & $0.72(4)$ \\
\hline \multirow[t]{3}{*}{$\mathrm{Li} 4$} & $4 \mathrm{c}$ & lit & - & - & - & - \\
\hline & & r.t. & 0 & 0 & $0.251(2)$ & $0.81(7)$ \\
\hline & & l.t. & 0 & 0 & $0.2510(16)$ & $0.77(6)$ \\
\hline \multirow[t]{3}{*}{ Ge1 } & $4 \mathrm{~d}$ & lit & 0 & $1 / 2$ & $0.6907(6)$ & $0.515(5)$ \\
\hline & & r.t. & 0 & $1 / 2$ & $0.69154(7)$ & 0.5 \\
\hline & & l.t. & 0 & $1 / 2$ & $0.69003(5)$ & 0.5 \\
\hline \multirow[t]{3}{*}{ P1 } & $4 \mathrm{~d}$ & lit & 0 & $1 / 2$ & $0.6907(6)$ & $0.485(5)$ \\
\hline & & r.t. & 0 & $1 / 2$ & $0.69154(7)$ & 0.5 \\
\hline & & 1.t. & 0 & $1 / 2$ & $0.69003(5)$ & 0.5 \\
\hline \multirow[t]{3}{*}{$\mathrm{P} 2$} & $2 \mathrm{~b}$ & lit & 0 & 0 & $1 / 2$ & 1 \\
\hline & & r.t. & 0 & 0 & $1 / 2$ & 1 \\
\hline & & l.t. & 0 & 0 & $1 / 2$ & 1 \\
\hline \multirow[t]{3}{*}{ S1 } & $8 \mathrm{~g}$ & lit & 0 & $0.184(2)$ & $0.410(2)$ & 1 \\
\hline & & r.t. & 0 & $0.18857(13)$ & $0.40707(10)$ & 1 \\
\hline & & 1.t. & 0 & $0.18954(11)$ & $0.40664(8)$ & 1 \\
\hline \multirow[t]{3}{*}{ S2 } & $8 \mathrm{~g}$ & lit & 0 & $0.299(2)$ & $0.095(2)$ & 1 \\
\hline & & r.t. & 0 & $0.29557(14)$ & $0.09727(9)$ & 1 \\
\hline & & 1.t. & 0 & $0.29440(12)$ & $0.09561(7)$ & 1 \\
\hline \multirow[t]{3}{*}{ S3 } & $8 g$ & lit & 0 & $0.699(2)$ & $0.791(2)$ & 1 \\
\hline & & r.t. & 0 & $0.69901(13)$ & $0.79168(9)$ & 1 \\
\hline & & l.t. & 0 & $0.69990(11)$ & $0.79051(7)$ & 1 \\
\hline
\end{tabular}

(ii) as a consequence, the occupancies of the Li positions are significantly different; (iii) the anisotropic displacement parameters for the Li sites also differ significantly ( $c f$. Table 3). The additional Li4 lies in a four-fold coordinated site which is located between the four channels along the $c$-axis (see Fig. 1). It connects two Li1 positions in adjacent channels with a reasonable jump distance of $2.9 \AA$ A Interestingly, this Li site shows - like the Li1 and Li3 positions - rather large anisotropic displacement parameters, see Table 3 . This can be interpreted as a sign that Li4 is active for diffusion (much more than the octahedrally coordinated Li2 site which shows much smaller thermal ellipsoids). While the thermal ellipsoids of Li1 and Li3 are roughly aligned along the $c$-axis (forming the diffusion channels), the thermal ellipsoid of Li4 is aligned perpendicular to the $c$-axis, thus forming diffusion pathways connecting the channels along the $c$-axis ( $c f$. Fig. 1A). This is in line with a recent study on the $\mathrm{Li}$ ion dynamics in the related material $\mathrm{Li}_{7} \mathrm{GePS}_{8}$ where no evidence of a strongly anisotropic diffusivity was observed. ${ }^{7}$ Note that a very similar Li4 site $(x=0, y=0, z \approx$ 0.22 ) has already been suggested by Adams and Rao from theoretical calculations of the Li site energy. ${ }^{8}$ In Fig. 1A, the crystal structure of LGPS as obtained from single crystal diffraction is displayed using thermal ellipsoids $(p=0.8)$. As a comparison, the structure as reported in the literature ${ }^{4}$ is shown in Fig. 1B (again with $p=0.8$ ). The largest difference occurs for the thermal ellipsoid of Li1 which shows an oblate shape in the 
Table 3 Anisotropic displacement parameters $\left(\AA^{2}\right)$ for $\mathrm{Li}_{10} \mathrm{GeP}_{2} \mathrm{~S}_{12}$ obtained from single crystal $\mathrm{X}$-ray diffraction at room temperature (r.t.) and $100 \mathrm{~K}$ (I.t.) in comparison with data obtained from neutron powder diffraction at room temperature (lit) ${ }^{4}$

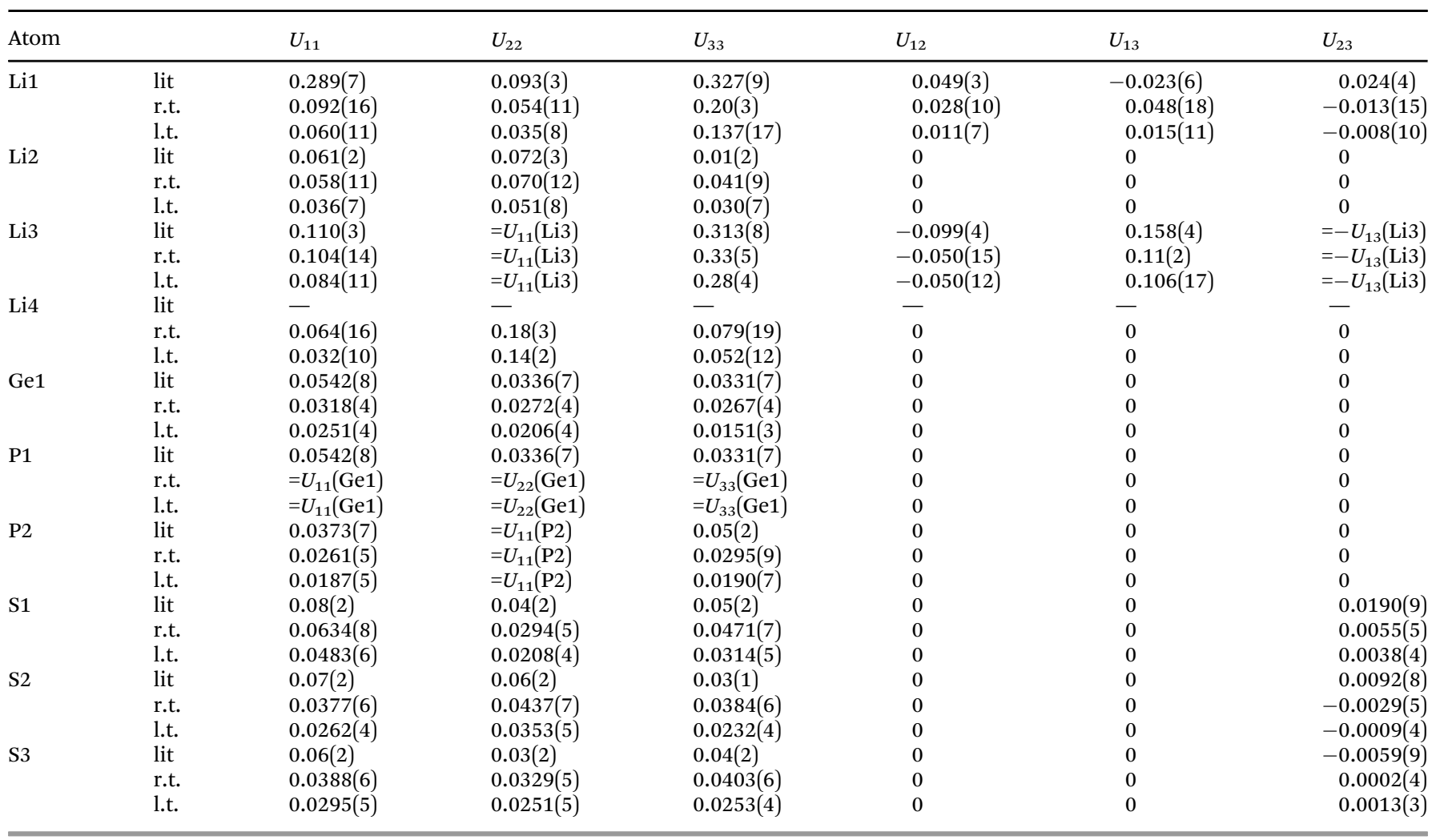

literature data and a prolate ellipsoid along the channels in our study. As to the Li occupancies, most importantly, the Li1 site should be discussed. The small distance of $1.6 \AA$ 方 between two adjacent Li1 sites makes an occupancy $>0.5$ rather improbable as already discussed by Adams and Rao. ${ }^{8}$ Therefore, the value obtained from single-crystal diffraction (0.48) is more realistic than the reported one (0.691). For comparison, the theoretical occupancy is 0.47 (ref. 8), in very good agreement with the experimental value. The higher occupancy of Li1, Li2, and Li3 in the literature probably results from neglecting the Li4 site. This evidently leads to higher occupancies for Li1, Li2, and Li3 to fulfill the stoichiometric amount of $\mathrm{Li}$ in the unit cell.

Concluding, the structure of $\mathrm{Li}_{10} \mathrm{GeP}_{2} \mathrm{~S}_{12}$ was determined with single crystal X-ray diffraction. The structural model obtained from neutron powder diffraction and Rietveld refinement was largely verified. However, the structure solution revealed a fourth Li site. The anisotropic displacement parameters of this Li site suggest that this position is active for diffusion and connects the 1D Li diffusion channels formed by Li1 and Li3. The potential landscape for $\mathrm{Li}$ in these diffusion pathways might be revealed by a temperature-dependent analysis of diffraction data using anharmonic potentials, in the future.

\section{Acknowledgements}

We thank Dr Jürgen Nuss for performing the single-crystal X-ray diffraction measurements.

\section{References}

1 J.-M. Tarascon and M. Armand, Nature, 2001, 414, 359.

2 R. Murugan, V. Thangadurai and W. Weppner, Angew. Chem., Int. Ed., 2007, 46, 7778.

3 H.-J. Deiseroth, S.-T. Kong, H. Eckert, J. Vannahme, C. Reiner, T. Zaiss and M. Schlosser, Angew. Chem., Int. Ed., 2008, 47, 755.

4 N. Kamaya, K. Homma, Y. Yamakawa, M. Hirayama, R. Kanno, M. Yonemura, T. Kamiyama, Y. Kato, S. Hama, K. Kawamoto and A. Mitsui, Nat. Mater., 2011, 10, 682.

5 Y. Mo, S. P. Ong and G. Ceder, Chem. Mater., 2012, 24, 15.

6 S. P. Ong, Y. Mo, W. D. Richards, L. Miara, H. S. Lee and G. Ceder, Energy Environ. Sci., 2013, 6, 148.

7 A. Kuhn, V. Duppel and B. V. Lotsch, submitted.

8 S. Adams and R. P. Rao, J. Mater. Chem., 2012, 22, 7687.

9 M. Xu, J. Ding and E. Ma, Appl. Phys. Lett., 2012, 101, 031901.

10 Further details of the crystal structure investigations may be obtained from Fachinformationszentrum Karlsruhe, 76344 Eggenstein-Leopoldshafen, Germany (fax: +49-7247-808666; e-mail: crysdata@fiz-karlsruhe.de, http://www.fiz-infor mationsdienste.de/en/DB/icsd/depot_anforderung.html) on quoting the deposition numbers given in Table 1 .

11 G. M. Sheldrick, Acta Crystallogr., Sect. A: Found. Crystallogr., 2007, 64, 112. 